\title{
Tuberculosis en el personal de salud del Servicio de Salud Metropolitano Sur de Santiago, Chile
}

\author{
Alberto Fica C., Paulina Ramonda C., M. Irene Jemenao P., Alejandra Zambrano G., Marcela Cifuentes D., \\ Naldy Febré V., M. Cristina Ajenjo H., Luis Delpiano M. y Alexis Diomedi P.
}

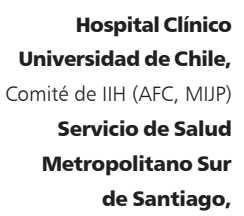

Programa de Tuberculosis (PRC)

Hospital Clínico Pontificia Universidad Católica de Chile, CPC-IAAS

(AZG, MCAH)

Complejo Hospitalario San

Borja Arriarán, Comité de IIH (MCD, LDM)

Clínica Las Condes Santiago,

Comité de IIH (NFV)

Hospital Del Salvador, Comité

Consultivo de Infecciones

Intrahospitalarias (ADP)

Recibido: 17 de enero 2008

Aceptado: 02 de diciembre 2008

Correspondencia a:

Alberto Fica C.

afica@redclinicauchile.cl

\section{Tuberculosis in health care workers from a public health service in Santiago, Chile}

Objective: To evaluate the risk of tuberculosis (TBC) among health care workers (HCW) of the Southern Metropolitan Health Service (SMHS) of Santiago, Chile. Method: A retrospective study using records of patients receiving TBC treatment in the SMHS from 2001 to 2006 was performed, in which HCW were identified. Total population of HCWs at risk was calculated using annual records of personnel hired at the SMHS. Data on TBC cases and rates were compared against data of the SMSH and hazard ratio (HR) and confidence intervals obtained. Results: Fourteen cases were identified, predominantly among auxiliary personnel (n: 4, 35.7\%), nursing staff and ambulance drivers (n: 2, 14.3\% each). Cases occurred in personnel from $41.7 \%$ of hospitals and $10.3 \%$ of ambulatory care centers within the SMHS and $92.2 \%$ involved personnel with direct patient care or contact. Pulmonary localization was seen in $11(78.6 \%)$, and more than half $(57.2 \%)$ had a positive sputum stain or culture. All cases initiated treatment, but 1 abandoned it and other died of liver failure associated to cirrhosis (7.1\% each). Between 2003 and 2006, the annual rate of TBC among HCW ranged between 0 and 79 per 100.000, and during 2004 it -10.62). Conclusions: Despite TBC rate decline in Chile, this disease still represents a significant occupational risk for HCW. Notably, more than half of cases among HCWs are contagious, and despite treatment, some have a lethal evolution.

Key words: Ttuberculosis, health care workers, occupational exposure, disease transmission.

Palabras clave: Tuberculosis, personal de salud, transmisión, riesgo ocupacional.

\section{Introducción}

$\mathrm{L}$ a tuberculosis (TBC) representa un riesgo ocupacional, tanto para los trabajadores de la salud como para los estudiantes de la salud ${ }^{1,2}$. Este riesgo se expresa como una infección asintomática (o TBC latente) cuando se asocia a una conversión de la prueba de tuberculina o como TBC clínica cuando existe un cuadro compatible con confirmación microbiológica en un paciente sintomático. La TBC clínica puede, en algunos casos, tener una evolución letal por inmunosupresión del afectado y/o por contagio con cepas multi-resistentes ${ }^{3}$. En poblaciones en las que no se contempla la vacuna BCG es posible efectuar estudios anuales de conversión tuberculínica para evaluar la tasa de nuevos funcionarios infectados por $M$. $t u$ berculosis. En países con alta cobertura de vacuna BCG como el nuestro, la población con una prueba inicial positiva es muy alta, lo que limita el contar con un grupo inicial negativo para el seguimiento. En estas condiciones, la estrategia alternativa ha sido reportar las tasas anuales de TBC clínica en el personal y comparar estos resultados con los de la población general. En el único trabajo publicado al respecto en Chile, Valenzuela y cols, describieron hacia mitad de la década anterior, un riesgo aproximadamente seis veces mayor respecto a la población general, de TBC clínica en el personal de salud (PS) de algunos hospitales escogidos en Santiago ${ }^{2}$.

Los objetivos de este trabajo fueron evaluar la tasa de prevalencia de TBC clínica observada en el PS del Servicio de Salud Metropolitano Sur (SSMS) de Santiago, analizar sus características y comparar este riesgo con el observado en la población general.

\section{Materiales y Métodos}

Escenario. Este trabajo se desarrolló en el Servicio de Salud Metropolitano Sur de Santiago (SSMS), instancia que tiene una población asignada de aproximadamente 1.150 .000 personas y cuenta con siete hospitales y 29 consultorios o centros de salud. El programa 
de TBC permite que todos los casos clínicos luego de su diagnóstico, sean derivados y atendidos en forma gratuita en los diferentes consultorios del SSMS, bajo la modalidad de terapia directamente observada. Los lugares de tratamiento están distribuidos en diferentes sitios de atención primaria u hospitales del SSMS.

Diseño del estudio. Para evaluar la magnitud de la TBC clínica en el PS se efectuó un estudio descriptivo transversal de prevalencia de enfermedad entre expuestos (PS) y no expuestos (resto de la población del SSMS). Este diseño fue escogido ya que no era posible asegurar que al comienzo del período de estudio todo el PS estuviera libre de enfermedad.

Definición de caso de TBC. Se consideró como caso de TBC todo aquel paciente o PS que ingresó durante el período de estudio al programa de TBC del SSMS, que trabajó y que además residía en la misma zona geográfica. Sólo se consideraron aquellos pacientes sin antecedentes de tratamiento. Debido a que la derivación es efectuada según el domicilio del paciente, en este trabajo sólo se incluyen personas que trabajaban en los centros de salud del SSMS y que al mismo tiempo vivían en esta zona.

Caracterización de los casos y período bajo estudio. En cada uno de los sitios de tratamiento existe un registro con el número de casos por año, las características demográficas del paciente, la ubicación anatómica de la TBC, si el caso es nuevo o si existe un antecedente de TBC previa, el esquema empleado en cada caso, y además datos sobre el cumplimiento del tratamiento. Estos antecedentes fueron recogidos para el análisis.

El período de estudio abarcó desde el año 2001 al 2006, enviándose a cada lugar de atención una encuesta requiriendo que informase si se había atendido para el período indicado cualquier PS de algunas de las dependencias que integran el SSMS.

El PS afectado fue contactado para aplicar una entrevista estructurada sobre sus funciones, atención de pacientes con $\mathrm{TBC}$, prácticas de protección personal y datos sobre inmunosupresión, previo consentimiento informado. El estudio fue autorizado por el Comité de Ética del SMSS.

Tasas de prevalencia y razón de riesgo. Para el cálculo de las tasas de prevalencia en el PS se utilizó como denominador el total de funcionarios de hospitales y consultorios del SMSS para cada año (cuando este dato estaba disponible). Esta información fue entregada por el SSMS e incluyó el total de funcionarios de los hospitales públicos en cualquier categoría profesional o no profesional y en los centros de atención primaria municipales. Cuando fue posible, se calcularon tasas para aquellos hospitales donde se observó más de un caso en el período. Las tasas de TBC del
SSMS y la población respectiva para cada año bajo estudio fueron provistas por la Dirección del Programa de TBC del SSMS.

Análisis estadístico. Las tasas de prevalencia de TBC en el PS y en el SSMS fueron divididas para obtener la razón de riesgo (RR) en cada año. Se descontaron los casos observados en el PS de aquellos casos registrados en el SSMS. La razón de riesgo fue calculada de acuerdo a fórmulas preestablecidas. ${ }^{4}$ Los intervalos de confianza fueron calculados con el apoyo del Programa StatCalc de EpiInfo version 6.0.

\section{Resultados}

Características generales. Catorce casos fueron identificados en el PS entre el año 2001 y 2006. La edad mediana fue 43 años (rango 24 a 69, rango intercuartil 25-75: 29). Veinticinco por ciento tenía $\geq 55$ años y $2 \geq$ 60 años (14,3\%). Hubo predominio de casos entre el sexo femenino (n: $11,78,6 \%$ ). Cerca del $93 \%$ era de nacionalidad chilena (n: $13 ; 92,9 \%$ ) y uno de nacionalidad peruana $(7,1 \%)$.

El grupo afectado predominante correspondió al de técnicos paramédicos $(35,7 \%)$, enfermeras (os) y conductores de ambulancia (14,3\% cada uno) (Tabla 1). Otros funcionarios del área clínica incluyeron un odontólogo, un médico y un auxiliar de servicio $(7,1 \%$ cada uno). Un sólo funcionario administrativo apareció afectado en esta serie $(7,1 \%)$. En otro caso no se contó con información sobre la actividad del PS. De esta manera se pudo comprobar que al menos $92,9 \%$ de los involucrados laboraba en el área clínica.

Los casos se presentaron tanto en funcionarios de hospitales como de consultorios (50 y $35,7 \%$ respectivamente, con dos casos sin antecedentes disponibles, Tabla 1). Un total de cuatro hospitales $(47,1 \%$ del total de hospitales) y tres consultorios $(10,3 \%$ del total de centros de atención primaria) aparecieron asociados a los casos. Los servicios de trabajo involucrados incluyeron al personal de servicios de medicina (n: $3 ; 21,4 \%$ ), consultorios (n: $5 ; 35,7 \%)$ u otras áreas del ámbito clínico en hospitales (n: $3 ; 21,4 \%$ ). (Tabla 1).

Localización y condición bacilifera. La forma pulmonar alcanzó $>70 \%$ de los casos y más de la mitad de los casos fueron bacilíferos ( 8 de $14 ; 57,2 \%$, Tabla 1 ). Tres de los 14 casos se diagnosticaron sobre bases empíricas, sin confirmación microbiológica $(21,4 \%$, Tabla 1). Uno de los casos tenía antecedentes de TBC y correspondió a una mujer de 70 años que presentó un cuadro pulmonar con radiografía sugerente y baja de peso, que fue tratado empíricamente como TBC y con antecedentes de esta misma enfermedad a los 10 años de edad, sin haber recibido tratamiento entonces.

Tasas de prevalencia de TBC en el personal de 
Tabla 1. Características generales de los casos de tuberculosis observados en el personal de salud del SSMS ( $n: 14)$

\begin{tabular}{|c|c|c|}
\hline Parámetro & $\mathbf{n}$ & $\%$ \\
\hline Sexo femenino & 11 & 78,6 \\
\hline \multicolumn{3}{|l|}{ Nacionalidad } \\
\hline Chilena & 13 & 92,9 \\
\hline Peruana & 1 & 7,1 \\
\hline \multicolumn{3}{|l|}{ Personal de salud } \\
\hline Técnico paramédico & 5 & 35,7 \\
\hline Chofer de ambulancia & 2 & 14,3 \\
\hline Enfermera & 2 & 14,3 \\
\hline Médico & 1 & 7,1 \\
\hline Odontólogo & 1 & 7,1 \\
\hline Auxiliar de servicio & 1 & 7,1 \\
\hline Administrativo & 1 & 7,1 \\
\hline Sin información & 1 & 7,1 \\
\hline \multicolumn{3}{|l|}{ Distribución } \\
\hline Hospitales & 7 & 50,0 \\
\hline Consultorios & 5 & 35,7 \\
\hline Sin información & 2 & 14,3 \\
\hline \multicolumn{3}{|c|}{ Servicio de trabajo del personal de salud afectado } \\
\hline Medicina & 3 & 21,4 \\
\hline Otros servicios clínicos en hospitales & 3 & 21,4 \\
\hline Atención en consultorio & 5 & 35,7 \\
\hline Sin información & 3 & 21,4 \\
\hline \multicolumn{3}{|l|}{ Localización } \\
\hline Pulmonar & 11 & 78,6 \\
\hline Extrapulmonar & 3 & 21,4 \\
\hline \multicolumn{3}{|l|}{ Diagnóstico } \\
\hline Frotis o cultivo positivo en vía aérea & 8 & 57,2 \\
\hline Sólo biopsia & 3 & 21,4 \\
\hline Sin confirmación & 3 & 21,4 \\
\hline
\end{tabular}

salud. Los casos de TBC ocurrieron en cada año bajo análisis (rango 1 a 6 casos por año). Diez de los 14 casos en el PS se concentraron en los tres últimos años bajo estudio (2004 al 2006, 71,4\%).

Las tasas de prevalencia de TBC clínica fueron calculadas sólo para los años 2003 a 2006 (cuatro años) ya que no había información completa disponible sobre la dotación de personal del SSMS en los años previos.

Para el período 2003-2006, la tasa de prevalencia de TBC para el PS en todas sus formas osciló entre 0 y 79 por 100.000 y en dos años fue superior al valor registrado para el resto del SSMS (2004 y 2006. (Tabla 2). El número anual de casos de TBC observados en el SSMS osciló en valores cercanos a los 200 casos (Tabla 2).

La tasa de prevalencia de TBC fue significativamente mayor en el PS respecto a la tasa de TBC observada en el SSMS durante el año 2004 (RR 4,56; IC95 1,83 a 10,62). El mayor riesgo observado ese año influyó en el valor RR observado para todo el período (Tabla 2). Al excluir los datos del año 2004, se pierde la significación estadística para todo el período (datos no mostrados).

Se observó más de un caso de TBC en el PS en dos hospitales y en un consultorio del SSMS durante el período 2001-2006. Estos casos acumulados se observaron en los hospitales El Pino (dos casos el 2004, tasa 306 por 100.000 para ese año), Lucio Córdova (un caso el 2001 y otro el 2002, tasas no calculable por no contar con dotación de personal para esos años) y en el Consultorio Paine (dos casos el 2006 con una tasa de 1.550 casos por 100.000$)$.

Resultado del tratamiento. Todos los pacientes afectados iniciaron tratamiento antituberculoso. Doce de ellos lo completaron de acuerdo a la normativa

Tabla 2. Tasa de prevalencia y razón de riesgo de TBC clínica en todas sus formas en el personal de salud y en el SSMS, 2003 al 2006

\begin{tabular}{|c|c|c|c|c|c|}
\hline & 2003 & 2004 & 2005 & 2006 & Período \\
\hline Casos en el personal & 2 & 6 & 0 & 4 & 12 \\
\hline Personal expuesto & 7.368 & 7.583 & 7.732 & 7.997 & 30.680 \\
\hline Tasa en el personal* & 27,1 & 79,1 & 0,0 & 50,0 & 39,1 \\
\hline Casos en SSMS & 214 & 201 & 210 & 205 & 830 \\
\hline Tasa SSMS* & 18,9 & 17,9 & 18,0 & 17,5 & 18,1 \\
\hline Razón de riesgo (RR) & 1,46 & 4,56 & 0,00 & 2,91 & 2,20 \\
\hline IC95 & $0,36-5,86$ & $1,83-10,62$ & No calculable & $0,91-8,92$ & $1,19-3,98$ \\
\hline
\end{tabular}


nacional y fueron dados de alta $(85,7 \%)$, uno lo abandonó y el restante falleció durante el tratamiento por insuficiencia hepática asociada a cirrosis alcohólica (7,1\% cada uno).

Otros antecedentes. Solamente siete de los 14 casos $(50 \%)$ pudieron ser ubicados y encuestados, impidiendo una adecuada caracterización sobre las medidas de protección personal, antecedentes de inmunosupresión o historia de contacto con un caso bacilífero. Un paciente declaró ser contacto de un paciente bacilífero pulmonar también incluido en esta serie. En los entrevistados no se detectaron usuarios de corticoesteroides (previo al inicio de la TBC) y ninguno declaró padecer de infección por VIH. Aunque no tabulado, en las preguntas abiertas de la entrevista, las personas afectadas declararon que el uso de mascarillas tipo N95 o de aislamientos individuales fue un hecho infrecuente durante la atención de pacientes con TBC.

\section{Discusión}

En ausencia de estudios de conversión tuberculínica, la comparación de tasas de TBC clínica entre el PS y la población general de una zona geográfica ha permitido estimar el riesgo ocupacional de TBC. ${ }^{5-7}$ En este trabajo se escogió esta estrategia y se logró demostrar que para uno de los años evaluados el riesgo de TBC clínica en el PS fue significativamente mayor que en la población que habita en la misma zona geográfica y con una razón de riesgo entre cuatro y cinco veces superior. Este hallazgo es concordante con los datos obtenidos en la literatura científica, incluyendo un estudio publicado previamente en Chile, hace ya una década. ${ }^{1,2}$ La recopilación de diferentes estudios de incidencia en países en vías de desarrollo ha permitido establecer que la TBC en el PS implica unos 25 a 5.361 casos adicionales por cada 100.000 personas $^{1}$.

No obstante, este mayor riesgo no fue pesquisado en todos los años bajo estudio. Las razones que explican esta intermitencia no son claras pero podrían indicar variaciones en el número de pacientes con TBC atendidos en cada centro, prácticas de protección locales, variaciones en la planta física o en procedimientos respiratorios, aspectos que no pudieron ser averiguados en este trabajo. El número de pacientes con TBC atendidos anualmente en un hospital es un factor determinante en el riesgo observado en el $\mathrm{PS}^{8}$. Este riesgo aumenta con más de 200 casos ingresados anualmente o con menos de 10 funcionarios por caso hospitalizado. Es posible que el bajo número de pacientes con TBC observado en el SSMS en los años bajo escrutinio ( $\sim 200$ por año), con su correspondiente fracción reducida de hospitalizados y bacilíferos, explique la baja exposición del PS al riesgo y su comportamiento intermitente. Otra lectura también es válida: a pesar de la declinación de la TBC en Chile, el PS sigue estando en riesgo ocupacional para esta enfermedad. No obstante, algunos estudios no han demostrado un mayor riesgo para el PS, notablemente en la experiencia de Hong Kong. ${ }^{5}$ Sin embargo, se debe recordar que por cada caso de TBC en el PS han ocurrido numerosos eventos de contagio con el desarrollo de una infección asintomática que puede progresar en el futuro a enfermedad clínica. De esta manera, la ausencia de un RR significativamente elevado no descarta que esté ocurriendo una alta tasa de casos de infección latente entre el PS.

A pesar de la limitación para caracterizar las tasas de prevalencia en todos los años del estudio, en nuestra investigación se demuestra que este riesgo está distribuido en hospitales y consultorios y no se concentra en un solo sitio, pudiendo alcanzar además cifras siderales en unos pocos centros. Asimismo, una fracción importante de los casos se presenta como TBC bacilífera pulmonar, perpetuando el ciclo de transmisión hacia otros compañeros de trabajo. Se debe destacar que los casos en el PS aparecen mayoritariamente ligados al área clínica, y además relacionados a diferentes funciones, posibilitando también la transmisión hacia pacientes en diferentes escenarios. Otro hallazgo importante, es que el PS no está exento de abandonos de tratamiento, recaídas o incluso letalidad por la enfermedad, ${ }^{9}$ aunque no asociada aparentemente a infección por VIH sino que a patologías más prevalentes en Chile como la cirrosis hepática.

En algunos estudios, la TBC observada en el PS se asocia a una población más joven que los casos observados en la población general. ${ }^{5}$ En esta casuística, algunos casos fueron observados en personas activas más allá de su edad de jubilación. Estos datos sugieren que el perfil de presentación en Chile también está moldeado por la permanencia del PS en el sitio de trabajo por razones económicas.

Los resultados de nuestro estudio no son se comparables con los reportados previamente por Valenzuela y cols, quienes evaluaron el riesgo en algunos hospitales seleccionados de diferentes Servicios de Salud, incluyendo un hospital dedicado a patología respiratoria y también un laboratorio de referencia de micobacterias. ${ }^{2}$ Por su diseño, los resultados probablemente sobrestiman el riesgo. En contraste, en esta investigación se evaluó un Servicio de Salud completo y por una mayor extensión de tiempo. No obstante, ambos estudias lograron demostrar un mayor riesgo en el PS.

Para disminuir este riesgo deben aplicarse una serie de medidas administrativas, de infraestructura y de 
protección del personal, las que son de lenta implementación en escenarios con escasez de recursos económicos. ${ }^{10}$ Varias de ellas están contempladas en el Programa Nacional de TBC y/o en el Programa de Control y Prevención de Infecciones Intrahospitalarias del Ministerio de Salud.

Los resultados de este trabajo tienen varias limitaciones y sólo permiten estimar el riesgo de TBC entre el PS. En primer lugar, no se pudo incluir a aquellos funcionarios de la salud del SSMS que tuvieron TBC pero que residían en otras zonas de la Región Metropolitana. La falta de un registro centralizado de TBC para el PS impide conocer apropiadamente este riesgo, una limitante ya resuelta en algunos países desarrollados. En segundo lugar, no todos los casos de TBC en el personal pueden ser adscritos a un contagio ocupacional, como ha sido aclarado por diferentes estudios moleculares en países desarrollados; sólo entre 32 y $42 \%$ de los casos en dichos estudios fueron adquiridos por razones laborales. ${ }^{11,12}$ En este estudio no fue posible contar con esta herramienta. En tercer lugar, no fue posible evaluar en forma exhaustiva si parte del personal tenía alguna condición de riesgo para progresión a TBC clínica como por ejemplo infección por VIH. En los pocos casos entrevistados no se detectaron usuarios de corticoterapia. Tampoco fue posible conseguir una caracterización detallada sobre las condiciones de trabajo del PS como por ejemplo sobre la aplicación de aislamientos individuales, uso de salas con presión negativa o infraestructuras adaptadas para la realización de fibro-broncoscopias o autopsias. Finalmente, no fue posible categorizar los lugares de trabajo del personal en mayor detalle debido a su movilidad.

Los resultados de este estudio indican que aún quedan problemas por resolver para disminuir el riesgo de TBC ocupacional en nuestro medio.

\section{Resumen}

Objetivo: Evaluar el riesgo de tuberculosis (TBC) clínica a través de un estudio retrospectivo sobre la razón de prevalencia de TBC entre el personal de salud (PS) del Servicio de Salud Metropolitano Sur de la Región Metropolitana (SSMS) y la población de la misma zona. Método: Los casos entre el PS y el resto del SSMS, junto a la población total y la dotación del PS, se obtuvieron de los registros existentes en el propio SSMS (2001-2006). Resultados: Se identificaron 14 casos, afectando predominantemente a técnicos paramédicos $(35,7 \%)$, enfermeros y conductores de ambulancia (14,3\% cada uno). El 92,9\% de los afectados laboraba en el área clínica. Los casos se presentaron entre el PS de hospitales y consultorios. Los afectados involucraban al $41,7 \%$ del total de hospitales y a $10,3 \%$ de los centros de atención primaria. La localización fue predominantemente pulmonar $(78,6 \%)$ y más de la mitad tenía frotis o cultivo de expectoración positivo $(57,2 \%)$. Todos los casos iniciaron tratamiento, 12 lo completaron con éxito $(85,7 \%)$, uno lo abandonó y el restante falleció por falla hepática asociada a cirrosis $(7,1 \%$ cada uno). La tasa entre el PS varió entre 0 y 79 casos por 100.000 y el año 2004 fue significativamente superior al valor registrado en el SSMS (razón de riesgo 4,56; $\mathrm{IC}_{95}: 1,83$ - 10,62). Conclusiones: A pesar de la declinación de la TBC en Chile, aún representa un riesgo ocupacional para el PS. Notablemente, más de la mitad de los casos son bacilíferos y algunos casos tienen una evolución letal.

\section{Referencias}

1.- Joshi R, Reingold A L, Menzies D, Pai M. Tuberculosis among health-care workers in low- and middle-income countries: a systematic review. Plos Med 2006; 3: 2376-91.

2.- Valenzuela M T, Lagos A, Schroeder F, O’Ryan L. Transmisión de la tuberculosis al personal de salud. Rev Chil Infect 1998; 15 $280-4$.

3.- Jereb J A, Klevens R M, Privett T D, Smith P J, Crawford J T, Sharp V L, et al. Tuberculosis in health care workers at a hospital with an outbreak of multidrugresistant Mycobacterium tuberculosis. Arch Intern Med 1995; 155: 854-9.

4.- Armitage P, Berry G. Métodos estadísticos en epidemiología. En: Estadística para la Investigación Biomédica. Ediciones Doyma, Barcelona, 1992, pp 534-68.

5.- Tam C M, Leung C C. Occupational tuberculosis: a review of the literature and the local situation. Hong Kong Med J 2006; 12: 448-55

6.- Hosoglu S, Tanrikulu A C, Dagli C, Akalin S. Tuberculosis among health care workers in a short working period. Amer J Infect Control 2005; 33: 23-6.

7.- Fica A, Cifuentes M, Ajenjo M C, Jemenao M I, Zambrano A, Febré N, et al. Tuberculosis en el personal de salud. Rev Chil Infect 2008; 25: 243-55.

8.- Menzies D, Fanning A, Yuan L, Fiztgerald M. Tuberculosis among health care workers. N Engl J Med 1995; 332: 92-8.
9.- Naidoo S, Jinabhai C C. TB in health care workers in KwaZulu-Natal, South Africa. Int J Lung Dis 2006; 10: 676-82.

10.- Center for Disease Control and Prevention. Guidelines for preventing the transmission of Mycobacterium tuberculosis in healthcare facilities. MMWR Morbid Mortal Wkly Rep 2005; 54 (RR17): 1-141.

11.- De Vries G, Sebek MM, Lambregts-van Weezenbeek CS. Healthcare workers with tuberculosis infected during work. Eur Respir J 2006; 28: $1216-21$.

12.- Ong A, Rudoy I, Gonzalez L C, Creasman J, Kawamura L M, Daley C L. Tuberculosis in healthcare workers: A molecular epidemiologic study in San Francisco. Infect Control Hosp Epidemiol 2006; 27: 453-8. 